Review

\title{
Molecular Mechanisms of Transcription Activation by Juvenile Hormone: A Critical Role for bHLH-PAS and Nuclear Receptor Proteins
}

\author{
Travis J. Bernardo ${ }^{1}$ and Edward B. Dubrovsky ${ }^{1,2, *}$ \\ 1 Department of Biology, Fordham University, Bronx, NY 10458, USA; \\ E-Mail: tbernardo@fordham.edu \\ 2 Center for Cancer, Genetic Diseases, and Gene Regulation, Fordham University, \\ Bronx, NY 10458, USA
}

* Author to whom correspondence should be addressed; E-Mail: dubrovsky@fordham.edu; Tel.: +1-718-817-3660; Fax: +1-718-817-3645.

Received: 21 February 2012; in revised form: 15 March 2012 / Accepted: 16 March 2012 / Published: 22 March 2012

\begin{abstract}
Juvenile hormone $(\mathrm{JH})$ is responsible for controlling many biological processes. In several insect species $\mathrm{JH}$ has been implicated as a key regulator of developmental timing, preventing the premature onset of metamorphosis during larval growth periods. However, the molecular basis of $\mathrm{JH}$ action is not well-understood. In this review, we highlight recent advances which demonstrate the importance of transcription factors from the bHLH-PAS and nuclear receptor families in mediating the response to JH.
\end{abstract}

Keywords: Drosophila melanogaster; juvenile hormone; nuclear receptor; bHLH-PAS; methoprene-tolerant; germ cell-expressed

\section{Introduction}

The insect life cycle is regulated by two major hormones whose balance determines the course of development. Pulses of 20-hydroxyecdysone (ecdysone) initiate each of the major developmental transitions, including both larval molting and metamorphosis [1], while $\mathrm{JH}$ is classically viewed as an antimetamorphic hormone [2]. According to this model, a high titer of $\mathrm{JH}$ in the early larval instars directs ecdysone to initiate molting, while the absence of $\mathrm{JH}$ during the final instar allows ecdysone to 
trigger the morphological changes of metamorphosis. The most recent support for this paradigm comes from studies in which manipulating the levels of $\mathrm{JH}$ prolongs larval development (increased JH titer) or induces premature metamorphosis (reduced JH titer) [3-5]. However, changes in JH titer appear to produce a stronger or weaker effect depending upon the species. In Lepidoptera and Coleoptera exogenously applied JH produces supernumerary larval instars [4-6] while in higher Diptera such as Drosophila melanogaster the effect of ectopic JH is less dramatic, as it does not prevent pupa formation but inhibits some metamorphic activities, including differentiation of the abdominal histoblasts [7] as well as premature remodeling and apoptosis of the larval fat body [8].

Although much attention has been given to understanding the mechanisms of action for both of these hormones, currently a great deal more is known about signaling by ecdysone than by JH. Ecdysone is a direct regulator of transcription and, like other steroid hormones acts through a nuclear receptor pathway. The ecdysone receptor is heterodimer of the nuclear receptors EcR and USP that binds to the ecdysone response element (EcRE) and activates gene expression when ecdysone is bound to the ligand pocket of EcR [1]. In contrast to the extensive knowledge of ecdysone signaling, the molecular mechanisms underlying $\mathrm{JH}$ function remain poorly understood. $\mathrm{JH}$ is pleiotropic and controls not only development but also sexual behavior, pheromone production, caste determination, diapause, migration, and the synthesis of female yolk proteins and male accessory gland proteins [9]. In regulating these diverse processes $\mathrm{JH}$ appears to utilize multiple pathways, some of which involve the activation of gene expression [10-12] while others are transcription-independent [13,14]. The multifunctional nature of JH has impeded the characterization of its signaling mechanisms.

Recently, there has been much progress in understanding the molecular basis of JH signaling. In this short review, we highlight significant advances that have been made in the past two years that demonstrate the importance of transcription factors, including basic-helix-loop-helix Per/Arnt/Sim (bHLH-PAS) and nuclear receptor proteins, in mediating the antimetamorphic activity of JH. We emphasize several unresolved issues, including how $\mathrm{JH}$ is integrated into the broader array of signaling pathways and the mechanisms which allow JH to operate distinctly in different insect species.

\section{MET as a JH Receptor}

Methoprene-tolerant (MET) was identified as a potential $\mathrm{JH}$ receptor through genetic studies in Drosophila, where Met null mutants fail to exhibit the morphogenetic defects that result from exogenous application of the JH analog methoprene [15]. MET homologs have since been identified in a wide range of insects, from holometabolans (summarized in [16]) to hemi- and ametabolous species [17]. Given the well-documented 'status quo' activity of JH in many insects, it was expected that mutation of the $\mathrm{JH}$ receptor would have severe consequences for larval development. This was confirmed in the red flour beetle Tribolium castaneum, where removal of Met expression produces a premature and lethal initiation of pupation [4]. In Drosophila, JH is essential to preadult development as its removal by genetic ablation of the corpora allata results in lethality around the time of head eversion and defects in larval development including precocious apoptosis of the fat body $[8,18]$. However, Met null mutants survive to adulthood and exhibit only minor defects in reproduction and in adult eye development $[18,19]$. One possible explanation for this paradox is that Drosophila possesses an additional Met-like gene, germ cell-expressed (gce) [20], in contrast to insects such as Tribolium that 
have only a single homolog [4]. However, until recently the function of GCE was poorly understood, and in light of the complexity of these observations the role of Drosophila MET as a JH receptor has remained controversial.

Studies of JH reception by MET have been scarce, and previously only a single report provided any direct evidence that Drosophila MET binds JH [21]. Recently, however, it was found that the ability of in vitro translated MET to bind $\mathrm{JH}$ is conserved among distantly related insects, including Drosophila, Tribolium, and the ametabolous Thermobia domestica [22]. Importantly, Tribolium MET binds JH with nanomolar affinity, an observation that is consistent with the requirement of MET for preventing premature metamorphosis in Tribolium [4] and for the induction of $\mathrm{JH}$ target genes including the antimetamorphic transcription factor Krüppel homolog $1(K r-h 1)$ [23,24]. Taken together, these data strongly indicate that in Tribolium, MET functions as a true $\mathrm{JH}$ receptor.

In Drosophila, the evidence concerning JH binding is less straightforward. The recent report found that both paralogs are capable of binding JH [22]. However, while MET was shown previously to bind JH with nanomolar affinity [21], these data have not been confirmed in an independent study and it is still not known whether JH is a high-affinity ligand for GCE. A comparative analysis of JH binding affinity for the Drosophila paralogs and other insect homologs of MET would help to clear up this issue. The need for a direct comparison is further highlighted by the observation that, despite having a strong affinity for JH [21] Drosophila MET was observed to exhibit substantially lower JH binding activity than either GCE or Tribolium MET [22]. It is unclear whether the discrepancy results from technical differences associated with the ligand-binding technique employed, but in any case it leaves open the question as to whether MET, GCE, or both proteins function as JH receptors in flies.

Despite the ambiguity of MET and GCE JH binding, several other lines of evidence support the idea that both paralogs do function similarly as JH receptors. First, MET and GCE are both able to mediate JH-dependent gene expression [25,26]. Second, while there are paralog-specific differences in gene structure and in the sequence of some protein motifs $[4,27,28]$ both paralogs exhibit comparable sequence identity to Tribolium and other homologs in their PAS domains (Table 1), with most of the divergence between MET and GCE apparently occurring in the C-terminus [28,29]. Finally, genetic studies provide a strong case that both proteins mediate $\mathrm{JH}$ signaling in vivo. Gce null mutants are resistant to exogenous $\mathrm{JH}$ and, although fully viable, display reduced fecundity [30]. The gce knockout thus phenocopies the Met null mutant. Another report found that RNAi-mediated reduction of gce expression is lethal, but it was argued that this comes from off-target effects of the dsRNA [19,30]. Nevertheless, in a Met null mutant background, overexpressed GCE restores the characteristic methoprene syndrome phenotype [19], and although the capacity for substitution is incomplete-GCE cannot restore oviposition or male sexual behavior in Met knockouts [19]-these findings suggest that both paralogs are capable of functioning in JH-dependent processes in vivo. Importantly, similar to JH-deficient larvae $[8,18]$ the Met/gce double knockout mutants fail to undergo head eversion, dying shortly after pupariation, and exhibit premature apoptosis in the larval fat body [30]. These observations demonstrate that MET and GCE are essential to preadult development in Drosophila but are functionally replaceable, confirming the belief that the viability of Met-deficient flies is attributed to redundancy between the paralogs. Moreover, the genetic studies lend support to the notion that MET and GCE function similarly to their Tribolium counterpart in acting as JH receptors in vivo. 
Table 1. Conservation of Drosophila Methoprene-tolerant (MET)/germ cell-expressed (GCE) sequence among insect homologs.

\begin{tabular}{|c|c|c|c|c|c|c|c|c|c|c|c|c|}
\hline \multirow[t]{2}{*}{ Homolog a } & \multicolumn{2}{|c|}{ bHLH } & \multicolumn{2}{|c|}{ PAS A motif } & \multicolumn{2}{|c|}{ PAS B motif } & \multicolumn{2}{|c|}{ PAC motif } & \multicolumn{2}{|c|}{ PAS Domain $1^{\text {b }}$} & \multicolumn{2}{|c|}{ Pas Domain 2} \\
\hline & MET & GCE & MET & GCE & MET & GCE & MET & GCE & MET & GCE & MET & GCE \\
\hline DmGCE & $82^{c}$ & - & 71 & - & 86 & - & 76 & - & 69 & - & 80 & - \\
\hline $\mathrm{BmMET}_{1}$ & 41 & 41 & 34 & 34 & 65 & 67 & 60 & 63 & 40 & 38 & 62 & 65 \\
\hline $\mathrm{BmMET}_{2}$ & 62 & 62 & 46 & 38 & 55 & 55 & 51 & 51 & 45 & 45 & 53 & 53 \\
\hline AaMET & 78 & 96 & 71 & 61 & 67 & 76 & 68 & 65 & 69 & 64 & 67 & 69 \\
\hline AgMET & 78 & 92 & 75 & 61 & 72 & 83 & 69 & 66 & 72 & 63 & 70 & 73 \\
\hline CpMET & 74 & 90 & 71 & 63 & 69 & 79 & 66 & 66 & 68 & 64 & 67 & 71 \\
\hline TcMET & 60 & 64 & 55 & 44 & 67 & 72 & 67 & 62 & 49 & 45 & 67 & 67 \\
\hline AmMET & 52 & 54 & 42 & 38 & 60 & 62 & 51 & 51 & 38 & 40 & 55 & 56 \\
\hline PaMET $^{\mathrm{d}}$ & 46 & 53 & 44 & 38 & 44 & 46 & 53 & 53 & 41 & 39 & 49 & 50 \\
\hline RpMET $^{\mathrm{d}}$ & 45 & 45 & 48 & 44 & 48 & 48 & 55 & 51 & 39 & 40 & 52 & 50 \\
\hline TdMET $^{\mathrm{d}}$ & 52 & 55 & 53 & 46 & 60 & 62 & 58 & 56 & 48 & 48 & 59 & 59 \\
\hline
\end{tabular}

${ }^{a}$ Dm, Drosophila melanogaster; Bm, Bombyx mori; Aa, Aedes aegypti; Ag, Anopheles gambiae; Cp, Culex pipiens; Tc, Tribolium castaneum; Am, Apis mellifera; Pa, Pyrrhocoris apterus; Rp, Rhodnius prolixus; Td, Thermobia domestica; ${ }^{\mathrm{b}}$ Residues comprising the complete PAS domains are described in [29]; ${ }^{\mathrm{c}}$ Numbers indicate percentage sequence identity; ${ }^{\mathrm{d}}$ Truncated bHLH domain lacks the N-terminal basic region.

\section{JH-Dependent Transcription Mediated by bHLH-PAS and Nuclear Receptor Proteins}

Analysis of $\mathrm{JH}$-inducible genes has identified a wide assortment of $\mathrm{JH}$-responsive sequences, suggesting that diverse elements may be involved in mediating or modulating JH-dependent transcription [2]. In general, though, the mechanism of JH-dependent transactivation by these elements is poorly understood. Often the proteins associated with a JH-responsive sequence are unidentified, or else have no known connection to in vivo JH function or even a known DNA-binding domain. However, recent efforts to characterize $\mathrm{JH}$ target genes have identified transcription factors and cognate response elements that are not only critical to JH-dependent transcription but are also members of the well-known bHLH-PAS or nuclear receptor protein families.

In Tribolium larvae, knockdown of Met prevents the expression of several JH-inducible genes, including the juvenile hormone esterase gene (jhe) and Krüppel homolog 1 (Kr-hl) [23,24]. An RNAi screen targeting other bHLH-PAS genes in Tribolium identified steroid receptor coactivator (TcSRC), the beetle homolog of the $\mathrm{NCoA} / \mathrm{SRC} / \mathrm{p} 160$ mammalian family of proteins (hereafter $\mathrm{NCoA}$ ), as an additional component required for JH-dependent transcription of $j h e$ and $K r-h 1$ [24]. Two other homologs of TcSRC-mosquito AaFISC and Drosophila Taiman-can also mediate the JH response. Taiman, AaFISC, and TcSRC each interact JH-dependently with MET through their PAS domains $[22,24,25]$. All three heterodimers are able to activate transcription by binding to a JH-responsive sequence identified upstream from the mosquito early trypsin gene. Not surprisingly, this $\mathrm{JH}$ response element (JHRE) contains an asymmetric E-box-like motif characteristic for the bHLH-PAS transcription factors [25]. These data suggest a possible mechanism for JH-dependent transcription in which $\mathrm{JH}$ stimulates MET to interact with its bHLH-PAS partner Taiman/AaFISC/TcSRC, and this heterodimer then binds to an E-box-like response element in JH target genes. So far the MET/AaFISC heterodimer was detected in vivo on the early trypsin gene [25], and it will be interesting to see whether other JH targets also contain an E-box-like element bound by a corresponding MET heterodimer. Of particular interest 
is $K r-h 1$, since it is critical to antimetamorphic action by JH and MET [2,17] and is a JH target gene not only in Tribolium [23] but also in Aedes aegypti [24,31], Drosophila [7,30], Bombyx mori [32] and the hemimetabolans Pyrrhocoris apterus [17] and Blatella germanica [33].

Another JH target, the Drosophila E75A gene, is in fact under dual hormonal regulation and can be activated by either ecdysone or JH [34]. Both responses are primary, as they do not require concurrent protein synthesis. Ecdysone activation is mediated by the EcR/USP heterodimer which binds to multiple EcREs distributed throughout the $30 \mathrm{~kb}$ region upstream from the $E 75 A$ transcription start site [35]. JH activation of this gene requires GCE but appears not to require MET, representing the first example of endogenous JH-dependent transcription mediated by GCE [26]. An RNAi screen against nuclear receptors found that $\mathrm{JH}$ activation of $E 75 \mathrm{~A}$ also requires an orphan receptor, FTZ-F1. Interestingly, the FTZ-F1 response element (F1RE) confers JH induction, as both MET and GCE can activate transcription of a reporter downstream of a DNA sequence containing F1REs (Figure 1). However, the precise role of FTZ-F1 in JH signaling is not understood, and it is unclear whether the F1RE is sufficient to mediate endogenous JH-dependent transcription. While heterodimerization with FTZ-F1 is clearly JH-dependent [29], MET and GCE mediate only weak JH-dependent transcriptional activation through the F1RE, suggesting that other proteins or DNA elements may also be involved in the JH response. Indeed, this possibility is consistent with the role of FTZ-F1 in ecdysone activation: in mosquitoes, FTZ-F1 facilitates ecdysone-dependent transcription by reinforcing DNA-binding of the ecdysone receptor to an ecdysone target gene containing both EcRE and F1RE sequences [36]. A similar mechanism may be at work for JH induction, whereby the F1RE operates in conjunction with other motifs such as the E-box-like response element. The identification of in vivo FTZ-F1 binding sites at several E75A enhancers [26] may eventually lead to additional sequences involved in JH-dependent transcription.

Figure 1. The F1RE is juvenile hormone (JH)-responsive. S2 cells were transfected with expression plasmids for FTZ-F1, MET, or GCE as indicated (x axis) as well as reporter containing ten copies of the FTZ-F1 response element. Luciferase activity normalized to constitutive $\beta$-galactosidase activity (y axis) was measured for samples treated with ethanol (light blue) or $5 \times 10^{-6} \mathrm{M}$ methoprene (dark blue) for $24 \mathrm{hrs}$. Asterisk indicates significant $(\mathrm{p}<0.05) \mathrm{JH}$-dependent activation. Heterodimer formation is required for activation, as seen by the FTZ-F1 ${ }^{\Delta \mathrm{H} 12}$ mutant which interacts poorly with MET and GCE [29]. Data are shown as the mean \pm S.D. from three independent experiments.

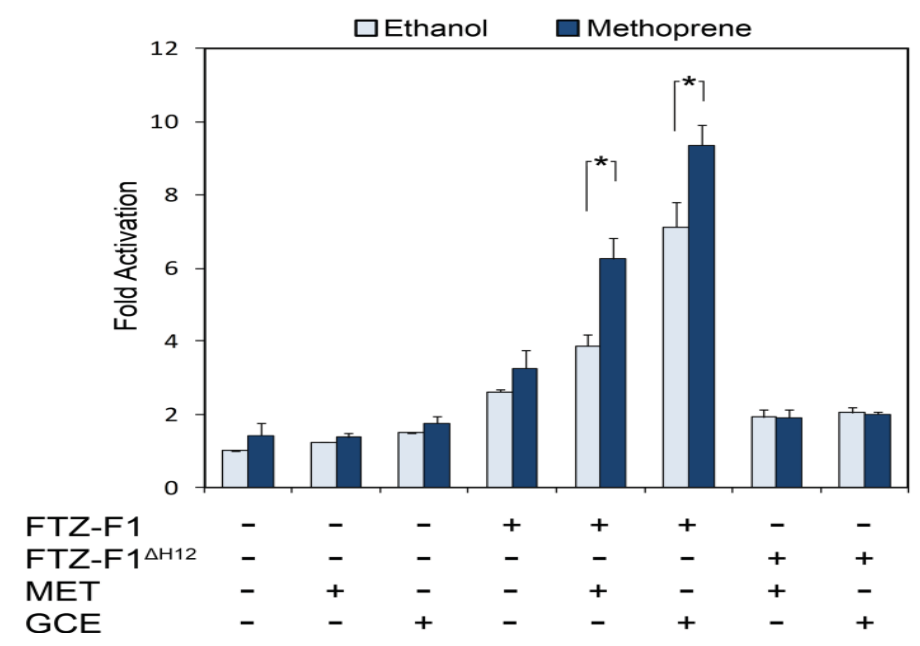




\section{Structural Elements of MET Involved in JH Reception and Function}

Consistent with its role as a $\mathrm{JH}$ receptor, MET possesses structures commonly involved in transcriptional regulation, including a bHLH and two PAS domains in its N-terminal half. The bHLH is a well-studied domain involved in partner dimerization and direct contact with DNA [36]. It is highly conserved among MET homologs [27,28] and adopts the classic helix-loop-helix conformation [29], a clear indication that this domain likely functions in a manner similar to other bHLH-PAS transcription factors. In contrast to bHLH, the structure of other regions of MET has until recently been poorly characterized. Several studies now provide additional information about the structure of MET, revealing features that are critical to its JH-dependent activity and establishing MET as a hormone receptor.

\subsection{Structure and Function of the PAS Domains.}

MET and GCE possess two conserved PAS motifs, PAS A and PAS B, that are a hallmark feature of bHLH-PAS proteins. PAS domains typically mediate protein-protein interactions, and for bHLH-PAS proteins participate in partner dimerization [37]. Deletions within either of the PAS motifs of MET disrupts dimerization with bHLH-PAS partners [25,38], indicating that both PAS domains are critical to the regulatory actions of MET.

Using a homology modeling approach [29], MET and GCE were shown to adopt two tandem canonical PAS folds, with a PAS motif comprising the N-terminal half of each fold. The C-terminal half of the second PAS fold is composed of a PAS-associated C-terminal (PAC) motif, as is often the case for PAS domains [39], while the C-terminal half of the first PAS fold is composed of discrete conserved sequences located between the PAS A and PAS B motifs. These sequences form secondary structural elements of the PAS fold in a manner analogous to the elements in a PAC motif, but they are separated by disordered loops whose presence significantly lengthens the region comprising the first PAS domain [29]. While the structure of the PAS domains awaits empirical determination, the models should aid future efforts to understand the molecular basis of bHLH-PAS partner formation by MET. Although a few critical residues have been identified in this respect $[22,38]$, a more comprehensive analysis has not been undertaken.

The presence of loops within the first PAS domain is intriguing, as loops are often essential to PAS-dependent signaling. For example, the Neurospora crassa photoreceptor Vivid is a small PAS protein whose flexible loop participates in binding the essential light-sensitive cofactor flavin [40]. Interestingly, the length and sequence of the PAS loops are highly variable among the insect homologs of MET [29], suggesting that they may provide functional diversity to this family of proteins. However, the precise role of these loops awaits further study.

PAS domains can function as signal sensors for a wide array of compounds [41], and their presence in MET supported the initial idea that MET might be a JH receptor. Indeed, the second PAS domain of Tribolium MET appears to be necessary and sufficient for JH binding, for JH-sensitive MET homodimerization, and for heterodimerization with the bHLH-PAS partner Taiman/AaFISC/ TcSRC [22]. Modeling of JH bound to this domain revealed several critical hydrophobic residues which, when mutated, block each of these $\mathrm{JH}$-dependent events, leading to the suggestion that MET 
uses its second PAS domain as a ligand binding domain (LBD) through which JH stimulates a conformational change from an inactive MET homodimer to an active heterodimer [22]. A critical test of this hypothesis would involve a comparison of empirically-derived LBD structures in the JH-bound and unbound states. Moreover, while comparative homology models (Figure 2) provide some support for the idea that Drosophila MET and GCE can bind JH in a manner similar to Tribolium MET, empirical structures would certainly help to resolve whether both Drosophila paralogs function as JH receptors.

Figure 2. Conserved structure of the MET ligand binding domain. Homology models of the second PAS domain for Tribolium castaneum MET (A) and Drosophila melanogaster GCE (B) and MET (C) were generated using HIF2 $\alpha$ (PDB ID 3F1P), showing that the overall domain structure is conserved. Residues shown above were implicated in $\mathrm{JH}$ binding for Tribolium MET [22] and are identical in Drosophila MET and GCE, except for a Ser substitution in GCE (arrow). An $\alpha$-helix containing NES-2 is shown in gold.
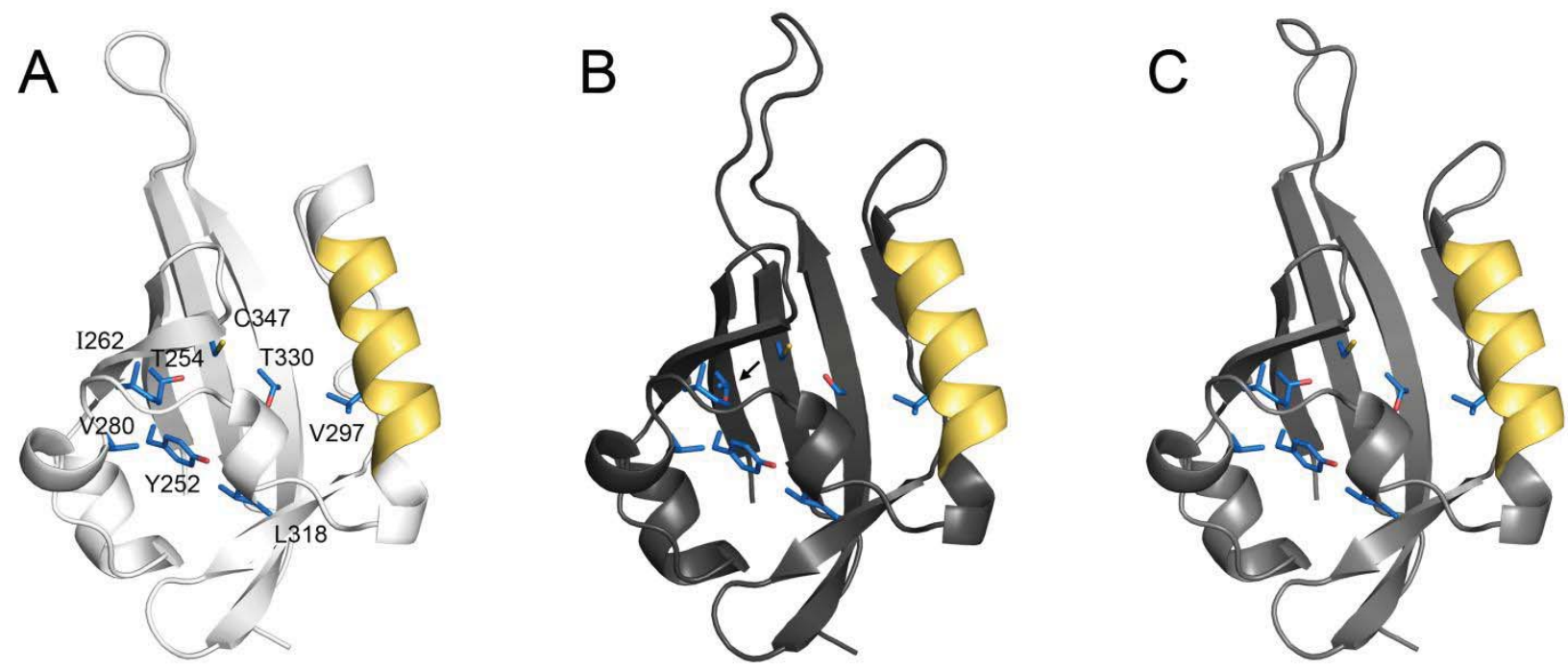

\subsection{Role of JH in MET Nuclear Localization.}

The ability to direct proteins into subcellular compartments is critical to cell function. For liganddependent transcription factors, translocation to and from the nucleus is often a dynamic process involving multiple nuclear localization (NLS) and export (NES) signals. Ligand often modifies the strength of these signals, thus influencing gene expression by determining the subcellular location of a protein [42]. The import/export signals in Drosophila MET were recently identified using ectopically expressed, YFP/CFP-tagged protein in mammalian cells [43]. MET possesses import and export motifs following the bHLH domain (NLS-1 and NES-1), within the second PAS domain (NLS-2 and NES-2), and an unidentified NES in the C-terminus. The presence of multiple NLS/NES motifs suggests that the localization of MET could be regulated dynamically, similar to other hormone-dependent transcription factors. However, Drosophila MET is exclusively nuclear both in cell culture [21,43] and in vivo [44]. This appears to result from the presence of NLS-1, which exhibits dominance over other motifs and forces JH-independent nuclear localization. In its absence, NES-2 in the second PAS domain causes MET to accumulate in the cytoplasm and enter the nucleus only in the presence of JH [43]. 
While the mechanism of JH-dependent nuclear entry is unclear, it is noteworthy that the $\alpha$-helix containing NES-2 participates in ligand binding by providing at least one critical residue [22] to the pocket (Figure 2). This suggests that JH may alter the activity of import/export signals in the second PAS domain by direct interference or by inducing a conformational change in the protein. Interestingly, the dominant NLS-1 of Drosophila MET is absent from other homologs, including GCE. This indicates that in Drosophila, MET has evolved as an exclusively nuclear protein while JH may play an important role in directing GCE to the nucleus. However, the extent to which JH-dependent subcellular localization occurs in GCE and in other insect homologs remains to be determined.

\subsection{NR Box in MET C-Terminus Enables JH-Dependent Interaction with Nuclear Receptor FTZ-F1}

While some bHLH-PAS proteins function as DNA-binding transcriptional activators, others operate through an entirely separate mechanism. bHLH-PAS proteins from the NCoA family do not dimerize through PAS-PAS interactions and lack the ability to bind DNA [45]. They instead serve as transcriptional coactivators, interacting with a nuclear receptor partner using a centrally located LxxLL (NR box) motif [46]. The NR box is an $\alpha$-helical structure that binds to activation function 2 (AF2), a hydrophobic binding groove in the LBD of nuclear receptors [47]. NCoA proteins typically possess several NR boxes, with each used to interact with a different group of nuclear receptors [46]. MET and GCE were recently found to possess a conserved motif C-terminal to the second PAS domain-LIxxL-that enables them to interact with the nuclear receptor FTZ-F1 [29]. This motif appears to function as a non-conventional NR box, as modeling of this motif in complex with FTZ-F1 shows a typical NR box/AF2 interaction with some mechanistic differences, such as the dispensability of the canonical charge clamp residues frequently used by nuclear receptors [47]. Similar to the NCoA proteins, MET thus appears to utilize an NR box to participate in signaling pathways through nuclear receptor interaction.

A crucial feature of MET that distinguishes it from the NCoA family, however, is that it acts as a ligand-binding protein. An important question therefore remains: what is the role of $\mathrm{JH}$ in regulating the activity of the MET NR box? For NCoA proteins, the NR box-containing region is sufficient for the interaction with nuclear receptors [48-50]. Moreover, the hormone-dependence of NCoA/nuclear receptor interactions is attributed to ligand binding by the nuclear receptor-constitutively active steroid receptors, including mammalian homologs of FTZ-F1, can bind NCoA proteins independently of hormone [51-54]. In contrast, the interactions of MET and GCE with FTZ-F1 are substantially enhanced by JH [29] and deletions in the second PAS domain disrupt the interaction with FTZ-F1 even when the NR box is intact (unpublished observations). This indicates that the NR box may only be functional when MET is in an active, JH-bound conformation.

Based on the recent studies of MET structure and function [22,29,43], we suggest a model for the role of $\mathrm{JH}$ in the regulatory activity of MET (Scheme 1). Binding of JH within the PAS domain triggers conformational changes that break the MET homodimer and promote important JH-dependent events including_interaction with bHLH-PAS proteins, interaction with nuclear receptors, and possibly nuclear localization. With its dependence on ligand in mediating interactions with both bHLH-PAS and nuclear receptor partners, MET thus occupies a unique position among the hormone receptors. 
Scheme 1. Model of MET activation through JH-dependent conformational changes. In the absence of $\mathrm{JH}$ (left), MET exists as a homodimer through interactions in the bHLH and PAS domains (gray). Binding of JH (right) stimulates an active conformation, promoting interaction with Taiman/AaFISC/TcSRC using the bHLH-PAS region, and with FTZ-F1 using a C-terminal NR box. JH may also influence the activity of nuclear export (NES) and import (NLS) signals in the ligand binding domain, promoting nuclear localization. Model is based primarily on evidence from $[22,29,43]$.

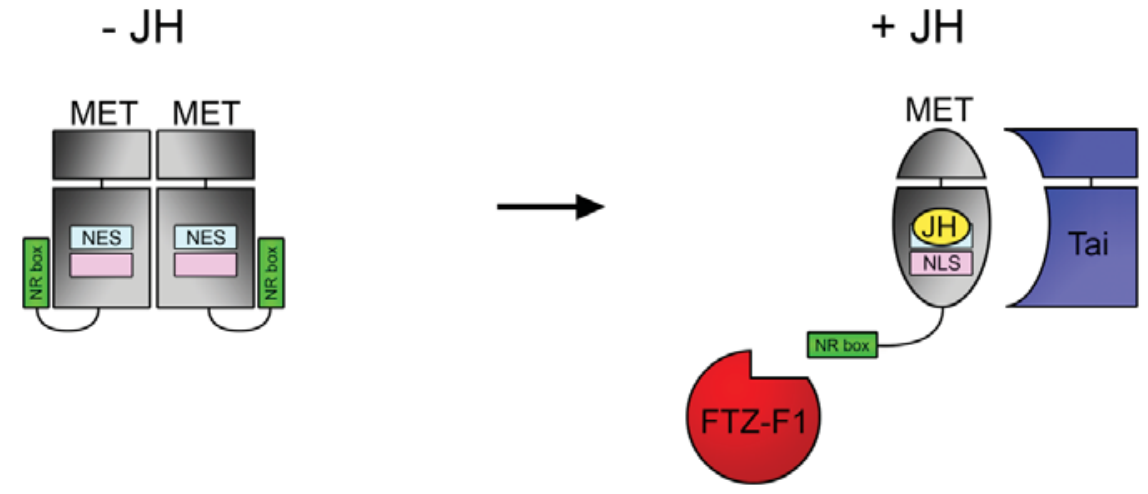

\section{Crosstalk between JH and Ecdysone}

Although the molecular mechanisms underlying JH action are gradually being elucidated, a critical question remains: what is the role of $\mathrm{JH}$ in the broader context of insect development? This question has grown increasingly complex with the observation that $\mathrm{JH}$ signaling involves more than one pathway $[13,14]$. Moreover, Jones recently demonstrated $[55,56]$ there are at least natural farnesoid hormones-methyl farnesoate, methyl epoxyfarnesoate (JH III), and methyl bisepoxyfarnesoate-involved in larval maturation and metamorphosis. An added complexity is the interplay between $\mathrm{JH}$ and other developmental signals, as JH interacts with the Target of rapamycin (TOR), Wingless (Wg), and TGF- $\beta$ pathways [57-59]. One of the most prominent examples of crosstalk involves the ecdysone signaling pathway: JH is thought to play a critical role in preadult development by altering the developmental outcome of early larval ecdysone pulses. While it is still not understood how these two hormones might interact during development, the recent identification of transcription factors involved in the $\mathrm{JH}$ response has provided some clues regarding possible mechanisms of JH/ecdysone crosstalk.

Expression of $K r-h 1$ is essential to preventing precocious metamorphosis in the early larval development of both holometabolous and hemimetabolous insects [17]. The JH/MET signaling pathway that activates $K r-h 1$ is thus an evolutionarily ancient strategy used to regulate the timing of larval development. In holometabolous insects, who transition to adulthood through a pupal stage, this pathway accomplishes its antimetamorphic function by repressing the expression of broad [17]. The broad gene is an ecdysone target activated at the onset of metamorphosis and is a critical pupal specifier: in Drosophila, misexpression of broad prematurely commits larvae to pupal development, while in its absence larvae develop normally but cannot pupariate [60]. Direct evidence for JH-dependent suppression of broad has been observed in Tribolium: removal of Met or $\mathrm{Kr}$ - $h 1$ results in precocious expression of broad accompanying precocious metamorphosis [23,61], while exogenous JH suppresses broad and thereby stimulates a supernumerary larval molt [62]. Until recently, it was 
unclear whether JH had a similar effect on broad expression in Drosophila. It has now been demonstrated in the larval fat body that genetic ablation of the CA or double knockout of Met and gce results in reduced $K r-h 1$ expression and precocious expression of broad concomitant with precocious apoptosis [30]. These findings suggest that at least in some larval tissues suppression of broad by the JH signaling pathway is critical to the timing of preadult development. It remains to be seen whether this phenomenon is a general feature of larval tissues.

In addition to repressing the ecdysone target broad, MET may also mediate crosstalk by physically interacting with components of the ecdysone signaling pathway. There have been several documented cases in which JH directly modifies the transcriptional response to ecdysone [63-65] and new evidence suggests that interactions between $\mathrm{JH}$ and ecdysone signaling proteins may play an important role in preadult development. Consistent with its homology to the NCoA family, Taiman/AaFISC/TcSRC functions not only as a JH-dependent interacting partner of MET [22,24,25] but also as an ecdysone receptor coactivator: it is critical to ecdysone-dependent regulatory processes in vivo and facilitates transcriptional activation by ecdysone $[36,66,67]$. Based on this observation, a model for crosstalk has been suggested [24,25] in which the use of Taiman/AaFISC/TcSRC by both hormones allows JH to antagonize ecdysone-dependent transcription by recruiting the shared protein to $\mathrm{JH}$ target genes. Antagonism through the use of shared transcription factors is a common mechanism of crosstalk between nuclear receptors as well as between nuclear receptors and bHLH-PAS proteins [68]. Ecdysone and $\mathrm{JH}$ could therefore coordinate their responses by competing for proteins involved in both signaling pathways, such as Taiman/AaFISC/TcSRC or FTZ-F1. The case of FTZ-F1 is particularly interesting in light of the observation that, during larval development, FTZ-F1 is briefly expressed prior to ecdysone pulses in the first two instars [69]. Since FTZ-F1 promotes metamorphosis by facilitating activation of the ecdysone genetic hierarchy [70], an active JH signaling pathway in larvae might prevent precocious metamorphosis in part by competing away FTZ-F1 from the ecdysone pathway. However, there is still no direct evidence that proteins involved in both ecdysone and $\mathrm{JH}$ signaling pathways contribute to crosstalk in vivo.

\section{Conclusions}

Significant advances have been made with respect to understanding the molecular mechanisms governing JH action. The evidence so far suggests that JH mediates its antimetamorphic function at the transcriptional level. By acting as a ligand for the bHLH-PAS transcription factor MET, JH stimulates an interaction between MET and partner proteins from the bHLH-PAS and nuclear receptor families, allowing MET to activate $\mathrm{JH}$ target genes containing E-box-like or FTZ-F1 response elements. Moreover, the involvement of $20 \mathrm{E}$ signaling components in the $\mathrm{JH}$ pathway could have implications for hormonal crosstalk. While it appears that both MET and GCE function as JH receptors, more detailed analyses of their $\mathrm{JH}$ binding, partner dimerization, nuclear localization, and transcriptional activation will illuminate the role of the JH signaling pathway in directing insect development.

\section{Acknowledgments}

This work was supported by National Science Foundation Grant 0653567 (to E.B.D.) and a Fordham University Graduate School of Arts and Sciences research fellowship (to T.J.B.). 


\section{References}

1. Riddiford, L.M.; Cherbas, P.; Truman, J.W. Ecdysone receptors and their biological actions. Vitam. Horm. 2000, 60, 1-73.

2. Riddiford, L.M. Juvenile hormone action: A 2007 perspective. J. Insect Physiol. 2008, 54, 895-901.

3. Tan, A.; Tanaka, H.; Tamura, T.; Shiotsuki, T. Precocious metamorphosis in transgenic silkworms overexpressing juvenile hormone esterase. Proc. Natl. Acad. Sci. USA 2005, 102, 11751-11756.

4. Konopova, B.; Jindra, M. Juvenile hormone resistance gene methoprene-tolerant controls entry into metamorphosis in the beetle tribolium castaneum. Proc. Natl. Acad. Sci. USA 2007, 104, 10488-10493.

5. Parthasarathy, R.; Tan, A.; Palli, S.R. bHLH-PAS family transcription factor methoprene-tolerant plays a key role in $\mathrm{JH}$ action in preventing the premature development of adult structures during larval-pupal metamorphosis. Mech. Dev. 2008, 125, 601-616.

6. Kremen, C.; Nijhout, H.F. Control of pupal commitment in the imaginal disks of precis coenia (lepidoptera: Nymphalidae). J. Insect Physiol. 1998, 44, 287-296.

7. Minakuchi, C.; Zhou, X.; Riddiford, L.M. Krüppel homolog 1 (kr-h1) mediates juvenile hormone action during metamorphosis of drosophila melanogaster. Mech. Dev. 2008, 125, 91-105.

8. Liu, Y.; Sheng, Z.; Liu, H.; Wen, D.; He, Q.; Wang, S.; Shao, W.; Jiang, R.J.; An, S.; Sun, Y.; et al. Juvenile hormone counteracts the bHLH-PAS transcription factors MET and GCE to prevent caspase-dependent programmed cell death in drosophila. Development 2009, 136, 2015-2025.

9. Flatt, T.; Tu, M.-P.; Tatar, M. Hormonal pleiotropy and the juvenile hormone regulation of drosophila development and life history. BioEssays 2005, 27, 999-1010.

10. Dubrovsky, E.B.; Dubrovskaya, V.A.; Bilderback, A.L.; Berger, E.M. The isolation of two juvenile hormone-inducible genes in drosophila melanogaster. Dev. Biol. 2000, 224, 486-495.

11. Beckstead, R.B.; Lam, G.; Thummel, C.S. Specific transcriptional responses to juvenile hormone and ecdysone in drosophila. Insect Biochem. Mol. Biol. 2007, 37, 570-578.

12. Willis, D.K.; Wang, J.; Lindholm, J.R.; Orth, A.; Goodman, W.G. Microarray analysis of juvenile hormone response in drosophila melanogaster S2 cells. J. Insect Sci. 2010, 10, art. no. 66.

13. Wheeler, D.E.; Nijhout, H.F. A perspective for understanding the modes of juvenile hormone action as a lipid signaling system. BioEssays 2003, 25, 994-1001.

14. Davey, K. From insect ovaries to sheep red blood cells: A tale of two hormones. J. Insect Physiol. 2007, 53, 1-10.

15. Wilson, T.G.; Fabian, J. A drosophila melanogaster mutant resistant to a chemical analog of juvenile hormone. Dev. Biol. 1986, 118, 190-201.

16. Li, Z.-Q.; Cheng, D.-J.; Wei, L.; Zhao, P.; Shu, X.; Tang, L.; Xiang, Z.-H.; Xia, Q.-Y. The silkworm homolog of methoprene-tolerant (met) gene reveals sequence conservation but function divergence. Insect Sci. 2010, 17, 313-324. 
17. Konopova, B.; Smykal, V.; Jindra, M. Common and distinct roles of juvenile hormone signaling genes in metamorphosis of holometabolous and hemimetabolous insects. PLoS One 2011, 6, e28728.

18. Riddiford, L.M.; Truman, J.W.; Mirth, C.K.; Shen, Y.-C. A role for juvenile hormone in the prepupal development of drosophila melanogaster. Development 2010, 137, 1117-1126.

19. Baumann, A.; Barry, J.; Wang, S.; Fujiwara, Y.; Wilson, T.G. Paralogous genes involved in juvenile hormone action in drosophila melanogaster. Genetics 2010, 185, 1327-1336.

20. Moore, A.W.; Barbel, S.; Jan, L.Y.; Jan, Y.N. A genomewide survey of basic helix-loop-helix factors in drosophila. Proc. Natl. Acad. Sci. USA 2000, 97, 10436-10441.

21. Miura, K.; Oda, M.; Makita, S.; Chinzei, Y. Characterization of the drosophila methoprene-tolerant gene product: Juvenile hormone binding and ligand-dependent gene regulation. FEBS J. 2005, 272, 1169-1178.

22. Charles, J.-P.; Iwema, T.; Epa, V.C.; Takaki, K.; Rynes, J.; Jindra, M. Ligand-binding properties of a juvenile hormone receptor. Proc. Natl. Acad. Sci. USA 2011, 108, 21128-21133.

23. Minakuchi, C.; Namiki, T.; Shinoda, T. Krüppel homolog 1, an early juvenile hormone-response gene downstream of methoprene-tolerant, mediates its anti-metamorphic action in the red flour beetle tribolium castaneum. Dev. Biol. 2009, 325, 341-350.

24. Zhang, Z.; Xu, J.; Sheng, Z.; Sui, Y.; Palli, S.R. Steroid receptor co-activator is required for juvenile hormone signal transduction through a bHLH-PAS transcription factor, methoprene tolerant. J. Biol. Chem. 2011, 286, 8437-8447.

25. Li, M.; Mead, E.A.; Zhu, J. Heterodimer of two bHLH-PAS proteins mediates juvenile hormoneinduced gene expression. Proc. Natl. Acad. Sci. USA 2011, 108, 638-643.

26. Dubrovsky, E.; Dubrovskaya, V.A.; Bernardo, T.J.; Otte, V.; DiFillipo, R.; Bryan, H. The Drosophila FTZ-F1 nuclear receptor mediates juvenile hormone activation of E75A gene expression through an intracellular pathway. J. Biol. Chem. 2011, 286, 33689-33700.

27. Wang, S.; Baumann, A.; Wilson, T.G. Drosophila melanogaster methoprene-tolerant (met) gene homologs from three mosquito species: Members of PAS transcriptional factor family. J. Insect Physiol. 2007, 53, 246-253.

28. Baumann, A.; Fujiwara, Y.; Wilson, T.G. Evolutionary divergence of the paralogs methoprene tolerant (met) and germ cell expressed (gce) within the genus drosophila. J. Insect Physiol. 2010, $56,1445-1455$.

29. Bernardo, T.J.; Dubrovsky, E.B. The Drosophila juvenile hormone receptor candidates Methoprene-tolerant (MET) and Germ cell-expressed (GCE) utilize a conserved LIxxL motif to bind the FTZ-F1 nuclear receptor. J. Biol. Chem. 2012, 287, 7821-7833.

30. Abdou, M.A.; He, Q.; Wen, D.; Zyaan, O.; Wang, J.; Xu, J.; Baumann, A.A.; Joseph, J.; Wilson, T.G.; Li, S.; et al. Drosophila met and gce are partially redundant in transducing juvenile hormone action. Insect Biochem. Mol. Biol. 2011, 41, 938-945.

31. Zhu, J.; Busche, J.M.; Zhang, X. Identification of juvenile hormone target genes in the adult female mosquitoes. Insect Biochem. Mol. Biol. 2010, 40, 23-29.

32. Wang, H.-B.; Ali, S.M.; Moriyama, M.; Iwanaga, M.; Kawasaki, H. 20-hydroxyecdysone and juvenile hormone analog prevent precocious metamorphosis in recessive trimolter mutants of bombyx mori. Insect Biochem. Mol. Biol. 2011, 42, 102-108. 
33. Lozano, J.; Belles, X. Conserved repressive function of Krüppel homolog 1 on insect metamorphosis in hemimetabolous and holometabolous species. Sci. Rep. 2011, 1, art. no. 163.

34. Dubrovsky, E.B.; Dubrovskaya, V.A.; Berger, E.M. Hormonal regulation and functional role of drosophila E75A orphan nuclear receptor in the juvenile hormone signaling pathway. Dev. Biol. 2004, 268, 258-270.

35. Bernardo, T.J.; Dubrovskaya, V.A.; Jannat, H.; Maughan, B.; Dubrovsky, E.B. Hormonal regulation of the E75 gene in drosophila: Identifying functional regulatory elements through computational and biological analysis. J. Mol. Biol. 2009, 387, 794-808.

36. Zhu, J.; Chen, L.; Sun, G.; Raikhel, A.S. The competence factor $\beta$ Ftz-F1 potentiates ecdysone receptor activity via recruiting a p160/SRC coactivator. Mol. Cell. Biol. 2006, 26, 9402-9412.

37. Kewley, R.J.; Whitelaw, M.L.; Chapman-Smith, A. The mammalian basic helix-loop-helix/PAS family of transcriptional regulators. Int. J. Biochem. Cell Biol. 2004, 36, 189-204.

38. Godlewski, J.; Wang, S.; Wilson, T.G. Interaction of bHLH-PAS proteins involved in juvenile hormone reception in Drosophila. Biochem. Biophys. Res. Commun. 2006, 342, 1305-1311.

39. Hefti, M.H.; Françoijs, K.J.; De Vries, S.C.; Dixon, R.; Vervoort, J. The PAS fold: A redefinition of the PAS domain based upon structural prediction. Eur. J. Biochem. 2004, 271, 1198-1208.

40. Zoltowski, B.D.; Schwerdtfeger, C.; Widom, J.; Loros, J.J.; Bilwes, A.M.; Dunlap, J.C.; Crane, B.R. Conformational switching in the fungal light sensor vivid. Science 2007, 316, 1054-1057.

41. Henry, J.T.; Crosson, S. Ligand-binding PAS domains in a genomic, cellular, and structural context. Annu. Rev. Microbiol. 2011, 65, 261-286.

42. Kumar, S.; Saradhi, M.; Chaturvedi, N.K.; Tyagi, R.K. Intracellular localization and nucleocytoplasmic trafficking of steroid receptors: An overview. Mol. Cell. Endocrinol. 2006, 246, 147-156.

43. Greb-Markiewicz, B.; Orlowski, M.; Dobrucki, J.; Ozyhar, A. Sequences that direct subcellular traffic of the drosophila methoprene-tolerant protein (MET) are located predominantly in the PAS domains. Mol. Cell. Endocrinol. 2011, 345, 16-26.

44. Pursley, S.; Ashok, M.; Wilson, T.G. Intracellular localization and tissue specificity of the methoprene-tolerant (met) gene product in drosophila melanogaster. Insect Biochem. Mol. Biol. 2000, 30, 839-845.

45. Partch, C.L.; Gardner, K.H. Coactivator recruitment: A new role for PAS domains in transcriptional regulation by the bHLH-PAS family. J. Cell. Physiol. 2010, 223, 553-557.

46. Leo, C.; Chen, J.D. The SRC family of nuclear receptor coactivators. Gene 2000, 245, 1-11.

47. Savkur, R.S.; Burris, T.P. The coactivator LXXLL nuclear receptor recognition motif. J. Pept. Res. 2004, 63, 207-212.

48. Hong, H.; Kohli, K.; Garabedian, M.J.; Stallcup, M.R. GRIP1, a transcriptional coactivator for the AF-2 transactivation domain of steroid, thyroid, retinoid, and vitamin D receptors. Mol. Cell Biol. 1997, 17, 2735-2744.

49. Onate, S.A.; Boonyaratanakornkit, V.; Spencer, T.E.; Tsai, S.Y.; Tsai, M.-J.; Edwards, D.P.; O'Malley, B.W. The steroid receptor coactivator-1 contains multiple receptor interacting and activation domains that cooperatively enhance the activation function 1 (AF1) and AF2 domains of steroid receptors. J. Biol. Chem. 1998, 273, 12101-12108. 
50. Voegel, J.J.; Heine, M.J.S.; Tini, M.; Vivat, V.; Chambon, P.; Gronemeyer, H. The coactivator TIF2 contains three nuclear receptor-binding motifs and mediates transactivation through CBP binding-dependent and -independent pathways. EMBO J. 1998, 17, 507-519.

51. Lazennec, G.; Ediger, T.R.; Petz, L.N.; Nardulli, A.M.; Katzenellenbogen, B.S. Mechanistic aspects of estrogen receptor activation probed with constitutively active estrogen receptors: Correlations with DNA and coregulator interactions and receptor conformational changes. Mol. Endocrinol. 1997, 11, 1375-1386.

52. White, R.; Sjöberg, M.; Kalkhoven, E.; Parker, M.G. Ligand-independent activation of the oestrogen receptor by mutation of a conserved tyrosine. EMBO J. 1997, 16, 1427-1435.

53. Ito, M., Yu, R.N.; Jameson, J.L. Steroidogenic factor-1 contains a carboxy-terminal transcriptional activation domain that interacts with steroid receptor coactivator-1. Mol. Endocrinol. 1998, 12, 290-301.

54. Xu, P.L.; Liu, Y.-Q.; Shan, S.-F.; Kong, Y.-Y.; Zhou, Q.; Li, M.; Ding, J.-P.; Xie, Y.-H.; Wang, Y. Molecular mechanism for the potentiation of the transcriptional activity of human liver receptor homolog 1 by steroid receptor coactivator-1. Mol. Endocrinol. 2004, 18, 1887-1905.

55. Jones, D.; Jones, G.; Teal, P.; Hammac, C.; Messmer, L.; Osborne, K.; Belgacem, Y.; Martin, J.R. Suppressed production of methyl farnesoid hormones yields developmental defects and lethality in drosophila larvae. Gen. Comp. Endocrinol. 2010, 165, 244-254.

56. Jones, G.; Jones, D.; Li, X.; Tang, L.; Ye, L.; Teal, P.; Riddiford, L.; Sandifer, C.; Borovsky, D.; Martin, J.-R. Activities of natural methyl farnesoids on pupariation and metamorphosis of drosophila melanogaster. J. Insect Physiol. 2010, 56, 1456-1464.

57. Edgar, B.A. How flies get their size: Genetics meets physiology. Nat. Rev. Genet. 2006, 7, 907-916.

58. Abdou, M.; Peng, C.; Huang, J.; Zyaan, O.; Wang, S.; Li, S.; Wang, J. Wnt signaling cross-talks with JH signaling by suppressing met and gce expression. PLoS One 2011, 6, e26772.

59. Huang, J.; Tian, L.; Peng, C.; Abdou, M.; Wen, D.; Wang, Y.; Li, S.; Wang, J. DPP-mediated TGF $\beta$ signaling regulates juvenile hormone biosynthesis by activating the expression of juvenile hormone acid methyltransferase. Development 2011, 138, 2283-2291.

60. Riddiford, L.M.; Hiruma, K.; Zhou, X.; Nelson, C.A. Insights into the molecular basis of the hormonal control of molting and metamorphosis from manduca sexta and drosophila melanogaster. Insect Biochem. Mol. Biol. 2003, 33, 1327-1338.

61. Konopova, B.; Jindra, M. Broad-complex acts downstream of met in juvenile hormone signaling to coordinate primitive holometabolan metamorphosis. Development 2008, 135, 559-568.

62. Suzuki, Y.; Truman, J.W.; Riddiford, L.M. The role of broad in the development of tribolium castaneum: Implications for the evolution of the holometabolous insect pupa. Development 2008, $135,569-577$.

63. Henrich, V.C.; Burns, E.; Yelverton, D.P.; Christensen, E.; Weinberger, C. Juvenile hormone potentiates ecdysone receptor-dependent transcription in a mammalian cell culture system. Insect Biochem. Mol. Biol. 2003, 33, 1239-1247.

64. Maki, A.; Sawatsubashi, S.; Ito, S.; Shirode, Y.; Suzuki, E.; Zhao, Y.; Yamagata, K.; Kouzmenko, A.; Takeyama, K.-I.; Kato, S. Juvenile hormones antagonize ecdysone actions through co-repressor recruitment to EcR/USP heterodimers. Biochem. Biophys. Res. Commun. 2004, 320, 262-267. 
65. Jones, G.; Jones, D.; Fang, F.; Xu, Y.; New, D.; Wu, W.-H. Juvenile hormone action through a defined enhancer motif to modulate ecdysteroid-activation of natural core promoters. Comp. Biochem. Physiol. B 2011, 161, 219-225.

66. Bai, J.; Uehara, Y.; Montell, D.J. Regulation of invasive cell behavior by taiman, a drosophila protein related to AIB1, a steroid receptor coactivator amplified in breast cancer. Cell 2000, 10, 1047-1058.

67. Bitra, K.; Tan, A.; Dowling, A.; Palli, S.R. Functional characterization of PAS and HES family bHLH transcription factors during the metamorphosis of the red flour beetle, tribolium castaneum. Gene 2009, 448, 74-87.

68. Swedenborg, E.; Rüegg, J.; Mäkelä, S.; Pongratz, I. Endocrine disruptive chemicals: Mechanisms of action and involvement in metabolic disorders. J. Mol. Endocrinol. 2009, 43, 1-10.

69. Yamada, M.-A.; Murata, T.; Hirose, S.; Lavorgna, G.; Suzuki, E.; Ueda, H. Temporally restricted expression of transcription and factor $\beta$ FTZ-F1: Significance for embryogenesis, molting and metamorphosis in drosophila melanogaster. Development 2000, 127, 5083-5092.

70. Broadus, J.; McCabe, J.R.; Endrizzi, B.; Thummel, C.S.; Woodard, C.T. The drosophila $\beta F T Z-F 1$ orphan nuclear receptor provides competence for stage-specific responses to the steroid hormone ecdysone. Mol. Cell 1999, 3, 143-149.

(C) 2012 by the authors; licensee MDPI, Basel, Switzerland. This article is an open access article distributed under the terms and conditions of the Creative Commons Attribution license (http://creativecommons.org/licenses/by/3.0/). 